\title{
Estudio de variabilidad geográfica de las hospitalizaciones potencialmente evitables en México durante tres quinquenios
}

\author{
Javier Valdés-Hernández ${ }^{1}$, Aldelmo Eloy Reyes-Pablo², Sonia Canún-Serrano ${ }^{3}$ y \\ Eduardo Navarrete-Hernández

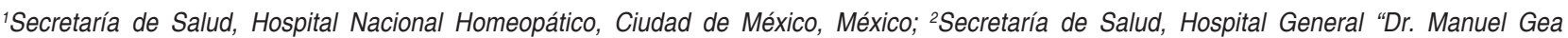

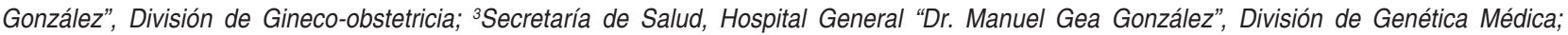 \\ ${ }^{4}$ Coordinación de Vigilancia Epidemiológica y Apoyo en Contingencias, Instituto Mexicano del Seguro Social. Ciudad de México, México
}

\begin{abstract}
Resumen
Introducción: Las enfermedades que motivan hospitalización potencialmente evitable tienen la característica de ser sensibles a la prevención, diagnóstico y control ambulatorio en atención primaria a la salud. Objetivos: Identificar la tendencia nacional de la hospitalización potencialmente evitable entre 2001 y 2015 y analizar el patrón geográfico a nivel municipal y priorizar municipios. Método: Se usaron los egresos hospitalarios de la Secretaría de Salud. Se calcularon tasas de prevalencia nacionales y razones municipales, estandarizadas por edad y sexo. Se emplearon estadísticos de variabilidad para analizar y elaborar mapas. Resultados: De los egresos hospitalarios, 10.39, 9.81 y $9.26 \%$ se clasificaron como hospitalizaciones potencialmente evitables para cada periodo quinquenal. La tasa nacional se incrementó en el lapso estudiado: de 36.27 a 47.24 por 10000 habitantes. La diabetes mellitus, las gastroenteritis y otras enfermedades de las vías respiratorias inferiores fueron las causas de mayor frecuencia. Los patrones geográficos en los tres periodos fueron semejantes. Se identificaron 487 municipios prioritarios, 174 con alto uso y 313 con sobreuso hospitalario, que concentraron $35.83 \%$ de las hospitalizaciones evitables, 8.58 y $27.25 \%$, respectivamente. Conclusiones: En México existe amplia variabilidad geográfica de la hospitalización potencialmente evitable con un patrón casi inmutable.
\end{abstract}

PALABRAS CLAVE: Hospitalizaciones evitables. Variabilidad geográfica. México.

\begin{abstract}
Introduction: Diseases that motivate potentially preventable hospitalization $(\mathrm{PH})$ have the characteristic of being sensitive to prevention, diagnosis and control on an outpatient basis in primary care. Objectives: To identify the national trend of potentially avoidable hospitalization between 2001 and 2015; to analyze its geographical pattern at the municipal level and prioritize municipalities. Method: Hospital discharge records from the Ministry of Health were used. National prevalence rates and municipal PH ratios, standardized by age and gender, were calculated. Variability statistics were used to analyze and generate maps. Results: Among all hospital discharges, $10.39 \%, 9.81 \%$ and $9.26 \%$ were classified as PH for each period. The national PH rate did increase in the studied period: from 36.27 to 47.24 per 10,000 population. Diabetes mellitus, gastroenteritis and other diseases of the lower respiratory tract were the most common causes. Geographic patterns of PH were similar for the three periods. A total of 487 priority municipalities were identified, 174 with hospital high use and 313 with overuse, $35.83 \%$ were avoidable hospitalizations, $8.58 \%$ and $27.25 \%$, respectively. Conclusions: In Mexico there is wide geographical variability in $\mathrm{PH}$, with an almost unchanging geographical pattern.
\end{abstract}

KEY WORDS: Avoidable hospitalizations. Geographic variability. Mexico.

Fecha de recepción: 29-07-2017

Fecha de aceptación: 23-09-2017

DOI://dx.doi.org/10.24875/GMM.17003613
Gac Med Mex. 2018;154:448-461

Disponible en PubMed

www.gacetamedicademexico.com 


\section{Introducción}

Las enfermedades que motivan una hospitalización potencialmente evitable tienen la característica de ser sensibles a la prevención, diagnóstico, tratamiento y control de manera ambulatoria en condiciones óptimas de atención primaria a la salud (APS); reciben el nombre de hospitalizaciones evitables (HE).

Las HE son un grupo de códigos de egresos hospitalarios de la Clasificación Internacional de Enfermedades (CIE-10) que representan los internamientos que se podrían haber evitado si la atención ambulatoria se hubiera prestado con calidad. ${ }^{1,2}$ Se han propuesto para evaluar el acceso a la APS, su calidad y capacidad para la prevención, diagnóstico temprano, tratamiento oportuno y seguimiento eficaz de las enfermedades sensibles al cuidado ambulatorio. Su validez y confiabilidad como indicador han sido evaluadas por diversos estudios. ${ }^{3,4}$ También se han empleado para la identificación de áreas prioritarias y la toma de decisiones en busca de fortalecer la APS y la evaluación de las acciones realizadas con ese propósito. ${ }^{5}$

El estudio de la variabilidad geográfica de la práctica médica, llevada a cabo en áreas pequeñas con el fin de romper el encubrimiento que ocurre en las investigaciones con agregaciones grandes, es una metodología que permite identificar con mayor certeza las áreas en las que ocurren $\mathrm{HE}$, con relativa independencia de los determinantes involucrados en la incidencia de las enfermedades, ya que emplea el lugar de residencia del paciente y no del hospital donde recibió la atención, considerando que el lugar donde viven los individuos es la sede desde la cual interactúan con los servicios de APS, en estado de salud o enfermedad. ${ }^{6}$

En México, en la evaluación del desempeño de los hospitales públicos de los años 2003 y 2011 de la Secretaría de Salud, entre otros aspectos se incluyó el análisis de las causas de $\mathrm{HE} ; ;^{7,8}$ en 2003 solo se incluyó la neumonía y en 2011 se integró un capitulado exclusivo en el que se analizó la frecuencia de internamientos por asma, gastroenteritis, deshidratación, angina de pecho, insuficiencia cardíaca congestiva, hipertensión arterial, enfermedad pulmonar obstructiva crónica y ciertos padecimientos odontológicos, lo que dio como resultado una tasa de HE de 29.6 por 10000 habitantes.

Un estudio de las HE en población del Instituto Mexicano del Seguro Social, patrocinado por el Banco Interamericano de Desarrollo, encontró que la tasa de HE basada en una lista de 20 causas mostró una tendencia descendente: de una tasa ajustada por edad de 87.5 por cada 10000 personas en 2001 pasó a 72.6 en $2009 .{ }^{9}$

Rodríguez Salgado et al., en su estudio de 2015 sobre HE por diabetes mellitus 2 en las tres principales instituciones del Sistema Nacional de Salud (Instituto Mexicano del Seguro Social, Instituto de Seguridad y Servicios Sociales de los Trabajadores del Estado y Secretaría de Salud) encontraron que la tasa de hospitalización por esta enfermedad a nivel nacional fue de 14 hospitalizaciones por cada 10000 habitantes $\geq 20$ años. Al considerar la condición de derechohabiencia, la tasa promedio de hospitalización para la población sin seguridad social fue de 15.3 y 12 para la población con seguridad social. ${ }^{10}$

El desglose de los datos en los estudios efectuados en México ha llegado a nivel nacional y estatal, sin embargo, los datos agregados en grandes áreas tienden a encubrir el comportamiento de los indicadores en áreas más concretas y no permiten identificar regiones prioritarias susceptibles para focalizar acciones correctivas. ${ }^{6}$ El presente estudio utilizó la metodología de la variabilidad geográfica para el análisis de las HE en tres periodos: 2001-2005, 2006-2010 y 2011-2015, tomando como referencia los datos de las unidades hospitalarias de la Secretaría de Salud.

Por otra parte, como fundamento para esperar escasa variabilidad en este indicador, hay que señalar que los servicios de salud públicos en México se encuentran organizados de forma piramidal: las unidades de primer nivel constituyen la base de la APS y desde ellas se llevan a cabo los distintos programas de prevención y control para las enfermedades más frecuentes en México, que resultan ser guías clínicas para su prevención, diagnóstico, tratamiento y control comunitario. Como ejemplos pueden mencionarse los programas de prevención y control de diabetes, hipertensión arterial, enfermedades diarreicas agudas e infecciones respiratorias agudas prevenibles por vacunación. Se han establecido pautas nacionales para el control de ciertos factores de riesgo: alimentación no saludable, inactividad física, alcoholismo, tabaquismo, por mencionar algunos. ${ }^{11}$ En este contexto puede considerarse que la estimación de HE apoyará a definir políticas de salud en México.

\section{Objetivos}

- Estimar la frecuencia y tendencia de las HE, en general y por causa, en tres quinquenios: 20012005, 2006-2010 y 2011-2015. 
- Analizar el patrón geográfico de las razones de hospitalizaciones potencialmente evitables a nivel municipal en los tres quinquenios de estudio.

- Priorizar los municipios con la finalidad de identificar zonas donde se podrían llevar a cabo intervenciones en salud.

\section{Método}

Se usaron los egresos hospitalarios de la Secretaría de Salud de 2001 a 2015, ${ }^{12}$ ya que de las bases de datos en este rubro disponibles al público por esta misma institución, solo en ellas se encuentra el registro de municipio de residencia habitual del paciente. Se agruparon en los tres quinquenios mencionados. Para la selección de HE se tomó la guía empleada por Rodríguez Abrego ${ }^{9}$ y otros estudios..$^{13}$ Debemos señalar que el asma, epilepsia, infección en el tracto urinario y del riñón, infección de la piel y del tejido subcutáneo, enfermedad inflamatoria de los órganos pélvicos femeninos y úlcera gástrica no están considerados en los programas de prevención y control prioritarios de la Secretaría de Salud, sin embargo, se encuentran guías clínicas sectoriales de algunas de estas enfermedades para su aplicación en el primer nivel de atención. ${ }^{14}$

Para calcular las razones de HE, estandarizadas por edad y sexo, se empleó el tamaño de la población por edad y sexo estimado por el Consejo Nacional de Población y se formaron siete grupos de edad, según el número de años: 0-4, 5-14, 15-24, 25-44, 45-64, $65-74$ y $\geq 75 .{ }^{15}$ Se prefirió usar el total de la población y no la población no asegurada como denominador, ya que un porcentaje importante de asegurados son usuarios de los servicios de la Secretaría de Salud. Se seleccionó el municipio como unidad de análisis por diferentes razones:

- Representa un mayor acercamiento a la residencia de los pacientes, lugar desde el cual los individuos se relacionan e interactúan con los servicios de APS, sobre todo en las causas de enfermedad sujetas al análisis que aquí se expone.

- Las patologías que requieren niveles sanitarios más complejos para su resolución trascienden la organización territorial de los servicios de salud a población abierta por problemas diversos, entre ellos, el sistema de referencia y contrarreferencia.

- El flujo de pacientes entre niveles de atención médica es independiente de la cobertura por hospital motivado por la oferta o preferencia de los usuarios.
- La organización de los servicios de atención médica a población abierta no está sujeta a una reglamentación territorial como la seguridad social, donde la residencia de los asegurados desempeña un papel importante para la asignación de las unidades médicas de correspondencia.

- Las regionalizaciones por jurisdicciones sanitarias o por hospitales son demarcaciones geográficas empleadas exclusivamente para la administración y planificación de los servicios gubernamentales.

En relación con el estudio del comportamiento de las tasas de HE por periodo y por causa a nivel nacional, este requirió el empleo del método de estandarización directo, tomando como referencia la suma de población de los tres quinquenios según edad y sexo.

Para estudiar la variabilidad geográfica de las HE a nivel municipal se utilizó el método de estandarización de tasas indirecto, en el que se tomó como media la tasa bruta de hospitalizaciones por edad y sexo a nivel nacional por cada periodo, a partir de las cuales se estimaron las razones de HE por municipio. En este análisis se estratificaron los municipios en función de las razones de $\mathrm{HE}$, enumerándolos del 1 al 5 : $\leq 0.50,0.80-0.51,0.81-1.19,1.20-1.49 \mathrm{y} \geq 1.50$.

Para clasificar los municipios según nivel de prioridad se empleó el método de clasificación probabilística ${ }^{16}$ de acuerdo con su frecuencia de ubicación en los estratos señalados, resultando cinco grupos: sobreuso, alto uso, uso medio, bajo uso e infrauso hospitalario. Los dos primeros representan las áreas de sobreutilización hospitalaria y se colorearon con color negro y gris oscuro en los mapas; los dos últimos grupos representan las regiones con subutilización del servicio hospitalario y se señalaron con azul cielo y verde, respectivamente. El grupo de uso medio se pintó de amarillo.

Se estimaron los estadísticos descriptivos generales para estudiar la variabilidad geográfica de las razones de HE (media, mediana, desviación estándar, varianza, valor mínimo y máximo, percentiles $1,5,10,20,25,50$, $75,80,90,95$ y 99). Para estudiar el grado de variabilidad de las razones de HE se obtuvieron las razones de variación de los percentiles (RVP) 1/99, 5/95, 10/90, $20 / 80$ y $25 / 75$. Por último, se estimó el componente sistemático de variación (CSV) para evaluar la varianza sistemática de las distribuciones de frecuencias en cada periodo, los valores cercanos a 1 fueron los esperados para el estimador; valores más bajos señalan que la incertidumbre o el azar se encuentra presente.

Para la elaboración de mapas se empleó el Mapa digital de México versión 1.6 y el Marco geoestadístico 2014 del Instituto Nacional de Estadística y Geografía (INEGI). ${ }^{17}$ 
a) Periodo 2001-2005

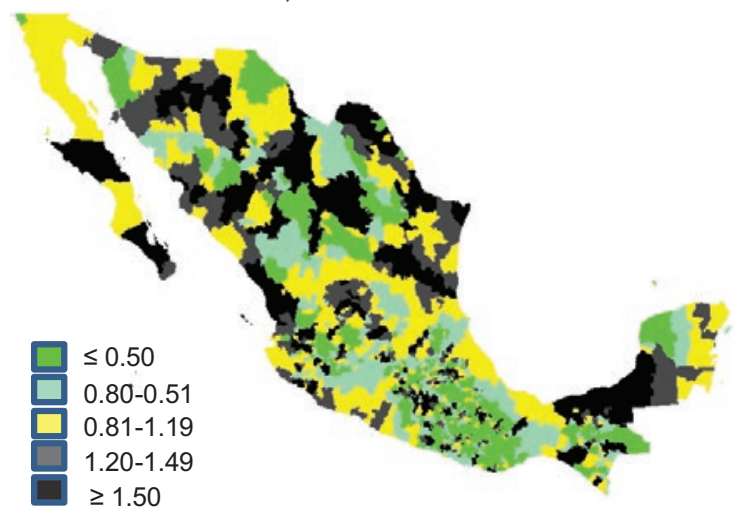

b) Periodo 2006-2010

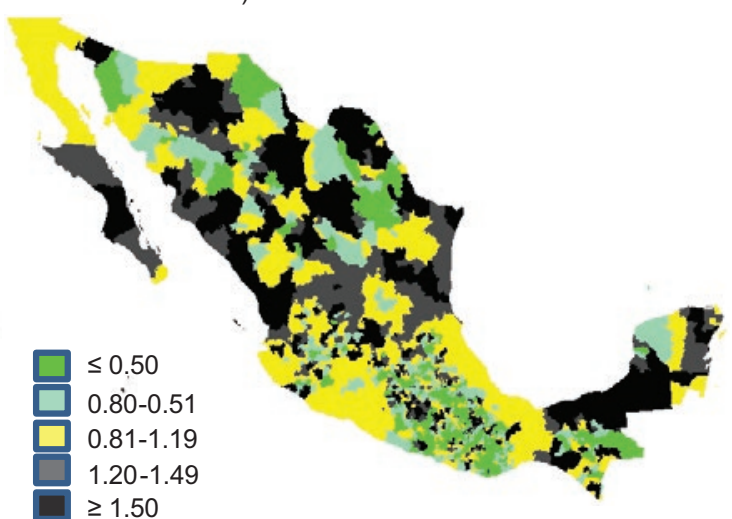

c) Periodo 2011-2015

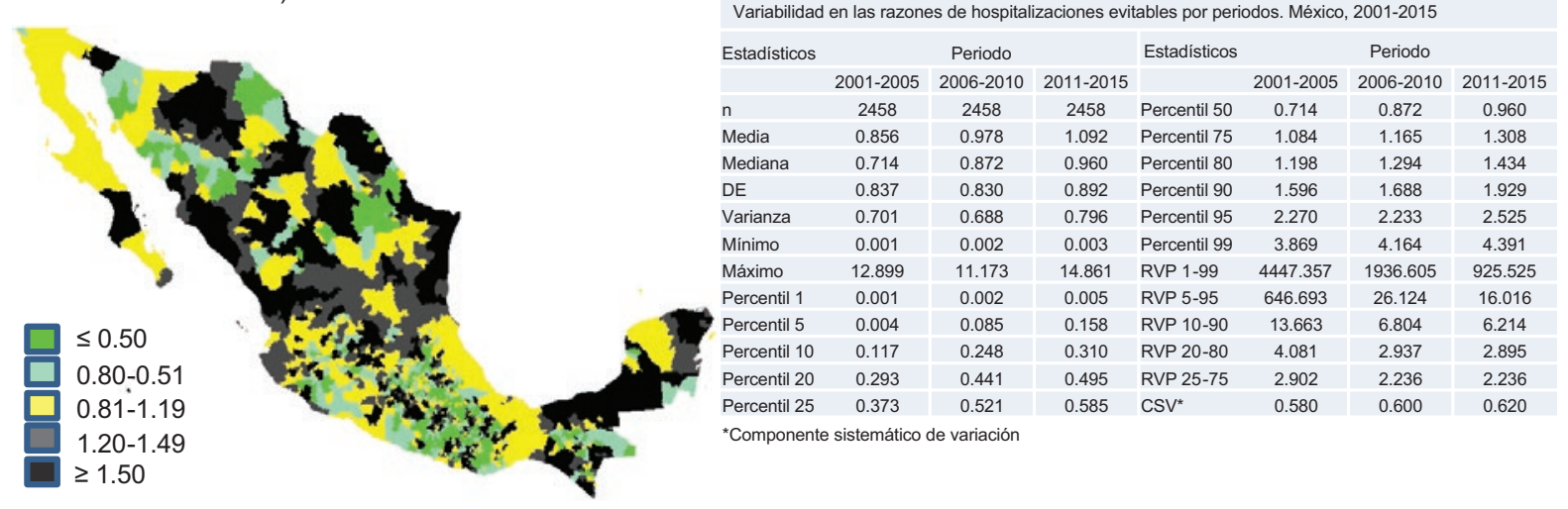

Figura 1. Patrones geográficos de las razones de hospitalizaciones evitables por municipio en tres quinquenios. México, 2001-2015.

La importancia relativa de las HE en cada periodo se obtuvo al tabular su proporción en referencia al total de egresos hospitalarios, así como su distribución porcentual por causas y grupos de edad (Tabla 1) y las tasas estandarizadas se obtuvieron con el método directo por edad y sexo de HE en función al total, causas y grupos de edad (Tabla 2).

Las distribuciones geográficas de las razones de HE por municipio estratificadas en cada quinquenio se ilustraron en mapas, en los que se aprecia el patrón geográfico asumido y el grado de dispersión de las cifras mediante los estimadores referidos (Figura 1). Con base en estas distribuciones geográficas y el método de clasificación probabilística se obtuvieron los municipios prioritarios que se muestran en la Figura 2; por último, se enlistaron los municipios prioritarios de acuerdo con su clave municipal otorgado por el INEGI (Tabla 3).

\section{Resultados}

Se estudiaron 35223193 egresos hospitalarios durante el periodo 2001-2015; $25 \%$ correspondió al primer quinquenio, $34 \%$ al segundo y $41 \%$ al tercero.
Del total de egresos hospitalarios, 10.39, 9.81 y $9.26 \%$ se clasificaron como HE para cada quinquenio, respectivamente (Tabla 1). Aunque proporcionalmente se apreció una leve disminución porcentual por estas causas de hospitalización, en números absolutos se incrementaron en promedio $14.23 \%$ durante el curso del lapso estudiado: 898 833, 1188416 y 1339 721 (Tabla 1).

Por causas de enfermedad, la diabetes mellitus, las gastroenteritis y otras enfermedades de las vías respiratorias inferiores fueron las de mayor porcentaje (total por periodo), llegando a constituir aproximadamente la mitad de las HE en cada quinquenio. Sin embargo, al observar la columna de crecimiento promedio en 12 causas se apreció incremento en su importancia relativa durante el lapso estudiado a costa de la disminución en las condiciones evitables, gastroenteritis, deficiencias nutricionales, infecciones en oídos, nariz y garganta, neumonía bacteriana, asma, otras enfermedades de las vías respiratorias inferiores e insuficiencia cardiaca.

Los grupos de edad 0-4, 25-44, 45-64 y $\geq 75$ años tuvieron mayor porcentaje de $\mathrm{HE}$ en cada quinquenio (total de HE). 
Gaceta Médica de México. 2018;154

Tabla 1. Total de hospitalizaciones evitables por periodo de estudio, causa y grupos de edad. México, 2001-2015

\begin{tabular}{|c|c|c|c|c|c|c|c|c|c|c|c|c|c|c|}
\hline \multirow{3}{*}{$\begin{array}{l}\text { Causas de hospitalizaciones } \\
\text { evitables }\end{array}$} & \multicolumn{9}{|c|}{ Quinquenio 2001-2005 } & \multirow{2}{*}{\multicolumn{4}{|c|}{$\begin{array}{r}\text { Quinquenio 2006-2010 } \\
\text { Grupos etarios }\end{array}$}} & \multirow{3}{*}{ 이 } \\
\hline & \multicolumn{7}{|c|}{ Grupos etarios } & \multicolumn{2}{|c|}{ Total } & & & & & \\
\hline & $0-4$ & $5-14$ & $15-24$ & $25-44$ & $45-64$ & $65-74$ & $\geq 75$ & $\mathrm{n}$ & $\%$ & $0-4$ & $5-14$ & $15-24$ & $25-44$ & \\
\hline $\begin{array}{l}\text { Enfermedades prevenibles por } \\
\text { vacunación }\end{array}$ & 43.99 & 8.65 & 10.70 & 18.41 & 11.56 & 3.99 & 2.69 & 4160 & 0.46 & 37.96 & 12.10 & 10.87 & 19.10 & $\frac{\frac{1}{2}}{\frac{1}{2}}$ \\
\hline Condiciones evitables & 6.96 & 7.54 & 13.27 & 31.22 & 26.00 & 8.64 & 6.36 & 17712 & 1.97 & 5.09 & 6.57 & 12.77 & 33.55 & \\
\hline Gastroenteritis y complicaciones & 53.09 & 14.53 & 6.17 & 10.45 & 7.84 & 3.53 & 4.39 & 155186 & 17.27 & 40.22 & 26.86 & 6.16 & 9.85 & : \\
\hline Anemia hipocrómica & 22.12 & 6.63 & 10.85 & 25.21 & 16.64 & 8.07 & 10.47 & 2626 & 0.29 & 10.55 & 9.44 & 9.99 & 25.66 & 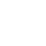 \\
\hline Deficiencias nutricionales & 58.13 & 12.39 & 3.25 & 5.25 & 5.92 & 4.52 & 10.52 & 10908 & 1.21 & 42.77 & 24.57 & 4.14 & 6.19 & $\frac{0}{\sigma}$ \\
\hline $\begin{array}{l}\text { Infecciones en oídos, nariz y } \\
\text { garganta }\end{array}$ & 47.14 & 25.36 & 9.22 & 11.43 & 4.26 & 1.22 & 1.36 & 33836 & 3.76 & 38.74 & 31.55 & 9.60 & 11.73 & \% \\
\hline Neumonía bacteriana & 39.76 & 9.14 & 4.21 & 10.36 & 11.86 & 8.72 & 15.94 & 26557 & 2.95 & 31.84 & 19.49 & 4.65 & 10.22 & $\frac{\pi}{2}$ \\
\hline Asma & 37.58 & 25.56 & 5.41 & 14.01 & 10.96 & 3.48 & 3.01 & 45608 & 5.07 & 35.53 & 31.66 & 5.18 & 12.66 & $\frac{5}{5}$ \\
\hline $\begin{array}{l}\text { Otras enfermedades de las vías } \\
\text { respiratorias inferiores }\end{array}$ & 47.85 & 6.25 & 1.02 & 3.02 & 10.29 & 11.97 & 19.60 & 93815 & 10.44 & 32.44 & 21.02 & 1.53 & 3.13 & $\stackrel{\complement}{\frac{\varrho}{c}}$ \\
\hline Hipertensión arterial & 0.18 & 0.50 & 2.08 & 15.05 & 39.24 & 21.18 & 21.75 & 33923 & 3.77 & 0.17 & 0.53 & 2.12 & 13.71 & $\frac{0}{\circ}$ \\
\hline Angina de pecho & 0.00 & 0.00 & 0.78 & 12.03 & 44.59 & 23.52 & 19.09 & 8581 & 0.95 & 0.00 & 0.00 & 0.79 & 10.02 & 肎 \\
\hline Insuficiencia cardiaca & 1.96 & 0.94 & 1.81 & 8.66 & 28.76 & 24.33 & 33.54 & 29807 & 3.32 & 1.48 & 1.22 & 1.81 & 8.91 & $\bar{c}$ \\
\hline Enfermedades cerebrovasculares & 0.53 & 0.66 & 1.76 & 8.70 & 27.70 & 23.66 & 36.99 & 45499 & 5.06 & 0.41 & 0.56 & 1.64 & 8.04 & 竧 \\
\hline Diabetes mellitus & 0.23 & 1.58 & 3.65 & 17.45 & 46.45 & 18.86 & 11.79 & 179433 & 19.96 & 0.19 & 1.49 & 3.28 & 16.61 & 은 \\
\hline Epilepsia & 22.26 & 18.74 & 17.70 & 27.60 & 9.57 & 2.30 & 1.82 & 22646 & 2.52 & 21.33 & 24.45 & 16.06 & 23.93 & $\frac{0}{\sigma}$ \\
\hline $\begin{array}{l}\text { Infección en el tracto urinario y } \\
\text { del riñón }\end{array}$ & 8.76 & 13.53 & 20.52 & 28.83 & 15.71 & 5.79 & 6.87 & 48377 & 5.38 & 8.91 & 15.43 & 19.27 & 25.88 & 을 \\
\hline $\begin{array}{l}\text { Infección de la piel y del tejido } \\
\text { subcutáneo }\end{array}$ & 12.96 & 16.58 & 12.43 & 25.82 & 21.29 & 6.01 & 4.91 & 37634 & 4.19 & 10.32 & 15.81 & 11.43 & 25.64 & 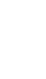 \\
\hline $\begin{array}{l}\text { Enfermedad inflamatoria de los } \\
\text { órganos pélvicos femeninos }\end{array}$ & 0.17 & 1.58 & 28.79 & 56.37 & 11.17 & 1.21 & 0.70 & 18319 & 2.04 & 0.26 & 1.50 & 26.66 & 56.59 & $\frac{0}{0}$ \\
\hline $\begin{array}{l}\text { Enfermedades relacionadas con } \\
\text { la atención prenatal y el parto }\end{array}$ & 0.99 & 1.61 & 65.80 & 31.46 & 0.14 & 0.00 & 0.00 & 40671 & 4.52 & 0.99 & 1.85 & 64.83 & 32.19 & 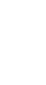 \\
\hline Úlcera gástrica & 1.34 & 1.52 & 4.07 & 23.46 & 35.56 & 15.25 & 18.80 & 43535 & 4.84 & 1.42 & 2.00 & 3.32 & 20.28 & 운 \\
\hline $\begin{array}{l}\text { Total de hospitalizaciones } \\
\text { evitables }\end{array}$ & 21.96 & 8.51 & 9.09 & 16.34 & 21.65 & 10.78 & 11.66 & 898833 & 100.00 & 15.16 & 12.26 & 9.85 & 16.14 & $\frac{1}{0}$ \\
\hline $\begin{array}{l}\% \text { de HE en relación con el total } \\
\text { de egresos }\end{array}$ & & & & & & & & 10.39 & & & & & & $\frac{\bar{c}}{E}$ \\
\hline Total de egresos hospitalarios & & & & & & & & 8649840 & & & & & & $\tilde{c}$ \\
\hline
\end{tabular}




\section{Total}

Grupos etarios

Total

$45-64 \quad 65-74 \geq 75$

n $\%$

\begin{tabular}{llllllllll}
\hline $0-4$ & $5-14$ & $15-24$ & $25-44$ & $45-64$ & $65-74$ & $\geq 75$ & & $n$ & $\%$
\end{tabular}

$13.30 \quad 3.80 \quad 2.87$

4397

0.37

$\begin{array}{lll}56.34 & 12.70 & 7.17\end{array}$

12.83

t.

\section{1}

6.

5

$\begin{array}{llll}6.29 & 4.41 & 11.63 & 10398\end{array}$

$\begin{array}{llll}5.41 & 1.37 \quad 1.59 \quad 3944\end{array}$

11.

10

\begin{tabular}{|c|c|c|c|c|c|c|c|c|c|c|c|c|c|c|}
\hline 37.59 & 21.95 & 23.93 & 51980 & 4.37 & 0.12 & 0.44 & 2.57 & 13.71 & 37.66 & 21.02 & 24.48 & 63597 & 4.75 & 7.94 \\
\hline 44.25 & 25.03 & 19.90 & 12155 & 1.02 & 0.00 & 0.00 & 0.55 & 9.73 & 46.01 & 23.98 & 19.73 & 14171 & 1.06 & 3.48 \\
\hline 29.39 & 24.10 & 33.10 & 38769 & 3.26 & 1.24 & 1.16 & 1.76 & 8.62 & 31.26 & 23.48 & 32.48 & 42508 & 3.17 & -1.46 \\
\hline 27.98 & 23.48 & 37.89 & 62515 & 5.26 & 0.31 & 0.48 & 1.40 & 7.32 & 28.93 & 23.54 & 38.02 & 73391 & 5.48 & 2.67 \\
\hline 47.25 & 18.69 & 12.49 & 261412 & 22.00 & 0.18 & 1.54 & 3.44 & 16.85 & 47.91 & 18.04 & 12.04 & 303597 & 22.66 & 4.32 \\
\hline 9.73 & 2.39 & 2.12 & 31801 & 2.68 & 21.31 & 25.67 & 15.82 & 21.89 & 10.63 & 2.53 & 2.15 & 41366 & 3.09 & 7.01 \\
\hline 16.42 & 6.40 & 7.69 & 69269 & 5.83 & 9.34 & 15.11 & 17.13 & 23.45 & 18.34 & 7.16 & 9.47 & 98045 & 7.32 & 10.79 \\
\hline 24.25 & 6.76 & 5.79 & 53628 & 4.51 & 9.93 & 14.10 & 10.82 & 25.89 & 26.00 & 7.33 & 5.93 & 68670 & 5.13 & 6.98 \\
\hline 12.81 & 1.24 & 0.93 & 22373 & 1.88 & 0.23 & 1.60 & 27.28 & 54.29 & 14.47 & 1.22 & 0.92 & 27334 & 2.04 & 0.04 \\
\hline 0.13 & 0.00 & 0.00 & 76106 & 6.40 & 0.46 & 1.84 & 65.48 & 32.08 & 0.13 & 0.00 & 0.00 & 106869 & 7.98 & 20.80 \\
\hline 35.51 & 16.11 & 21.36 & 58812 & 4.95 & 1.40 & 1.84 & 3.15 & 18.96 & 36.72 & 16.20 & 21.72 & 70757 & 5.28 & 2.93 \\
\hline 22.87 & 11.23 & 12.49 & 1188416 & 100.00 & 13.05 & 10.91 & 10.93 & 16.69 & 24.26 & 11.25 & 12.91 & 1339721 & 100.00 & 14.23 \\
\hline
\end{tabular}


Tabla 2. Tasa estandarizada por sexo y edad de hospitalizaciones evitables por 10000 habitantes según periodo de estudio, causa y grupos de edad. México, 2001-2015

\begin{tabular}{|c|c|c|c|c|c|c|c|c|c|c|c|c|c|}
\hline \multirow{3}{*}{$\begin{array}{l}\text { Causas de } \\
\text { hospitalizaciones } \\
\text { evitables }\end{array}$} & \multicolumn{8}{|c|}{ Quinquenio 2001-2005 } & \multicolumn{4}{|c|}{ Quinquenio 2006-2010 } & \multirow{2}{*}{$\frac{\infty}{2}$} \\
\hline & \multicolumn{7}{|c|}{ Grupos etarios } & \multirow[t]{2}{*}{ Total } & \multicolumn{4}{|c|}{ Grupos etarios } & \\
\hline & $0-4$ & $5-14$ & $15-24$ & $25-44$ & $45-64$ & $65-74$ & $\geq 75$ & & $0-4$ & $5-14$ & $15-24$ & $25-44$ & ¿ \\
\hline $\begin{array}{l}\text { Enfermedades } \\
\text { prevenibles por } \\
\text { vacunación }\end{array}$ & 0.06 & 0.01 & 0.02 & 0.03 & 0.02 & 0.01 & 0.00 & 0.16 & 0.06 & 0.02 & 0.02 & 0.03 & $\frac{\frac{\xi}{d}}{\frac{1}{4}}$ \\
\hline Condiciones evitables & 0.04 & 0.05 & 0.09 & 0.22 & 0.21 & 0.07 & 0.05 & 0.73 & 0.04 & 0.04 & 0.09 & 0.23 & $\dot{\varepsilon}$ \\
\hline $\begin{array}{l}\text { Gastroenteritis y } \\
\text { complicaciones }\end{array}$ & 2.79 & 0.82 & 0.37 & 0.65 & 0.55 & 0.24 & 0.30 & 5.73 & 2.62 & 1.70 & 0.39 & 0.62 & $\frac{.0}{0}$ \\
\hline Anemia hipocrómica & 0.02 & 0.01 & 0.01 & 0.03 & 0.02 & 0.01 & 0.01 & 0.10 & 0.01 & 0.01 & 0.01 & 0.03 & $\frac{6}{6}$ \\
\hline $\begin{array}{l}\text { Deficiencias } \\
\text { nutricionales }\end{array}$ & 0.21 & 0.05 & 0.01 & 0.02 & 0.03 & 0.02 & 0.05 & 0.40 & 0.17 & 0.10 & 0.02 & 0.02 & $\frac{1}{0}$ \\
\hline $\begin{array}{l}\text { Infecciones en oídos, } \\
\text { nariz y garganta }\end{array}$ & 0.54 & 0.31 & 0.12 & 0.15 & 0.07 & 0.02 & 0.02 & 1.23 & 0.59 & 0.46 & 0.14 & 0.17 & $\frac{\frac{1}{\pi}}{\frac{1}{\sigma}}$ \\
\hline Neumonía bacteriana & 0.36 & 0.09 & 0.04 & 0.11 & 0.14 & 0.10 & 0.19 & 1.03 & 0.32 & 0.19 & 0.05 & 0.10 & 앋 \\
\hline Asma & 0.58 & 0.42 & 0.09 & 0.25 & 0.23 & 0.07 & 0.06 & 1.71 & 0.73 & 0.63 & 0.10 & 0.25 & $\stackrel{c}{\varrho}$ \\
\hline $\begin{array}{l}\text { Otras enfermedades } \\
\text { de las vías respiratorias } \\
\text { inferiores }\end{array}$ & 1.52 & 0.21 & 0.04 & 0.11 & 0.44 & 0.50 & 0.82 & 3.64 & 1.54 & 0.98 & 0.07 & 0.14 & $\frac{\pi}{\frac{0}{2}}$ \\
\hline Hipertensión arterial & 0.00 & 0.01 & 0.03 & 0.20 & 0.60 & 0.32 & 0.32 & 1.49 & 0.00 & 0.01 & 0.04 & 0.27 & $\bar{c}$ \\
\hline Angina de pecho & 0.00 & 0.00 & 0.00 & 0.04 & 0.17 & 0.09 & 0.07 & 0.38 & 0.00 & 0.00 & 0.00 & 0.05 & $\frac{3}{3}$ \\
\hline Insuficiencia cardiaca & 0.02 & 0.01 & 0.02 & 0.10 & 0.39 & 0.32 & 0.44 & 1.31 & 0.02 & 0.02 & 0.03 & 0.13 & 응 \\
\hline $\begin{array}{l}\text { Enfermedades } \\
\text { cerebrovasculares }\end{array}$ & 0.01 & 0.01 & 0.03 & 0.16 & 0.57 & 0.48 & 0.74 & 2.01 & 0.01 & 0.01 & 0.04 & 0.19 & $\frac{8}{8}$ \\
\hline Diabetes mellitus & 0.01 & 0.10 & 0.25 & 1.25 & 3.79 & 1.51 & 0.93 & 7.85 & 0.02 & 0.14 & 0.32 & 1.62 & 응 \\
\hline Epilepsia & 0.17 & 0.15 & 0.15 & 0.25 & 0.10 & 0.02 & 0.02 & 0.87 & 0.26 & 0.29 & 0.19 & 0.28 & o \\
\hline $\begin{array}{l}\text { Infección en el tracto } \\
\text { urinario y del riñón }\end{array}$ & 0.14 & 0.24 & 0.38 & 0.56 & 0.34 & 0.12 & 0.15 & 1.93 & 0.23 & 0.40 & 0.50 & 0.66 & $\frac{8}{\frac{9}{0}}$ \\
\hline $\begin{array}{l}\text { Infección de la piel y } \\
\text { del tejido subcutáneo }\end{array}$ & 0.17 & 0.23 & 0.18 & 0.39 & 0.37 & 0.10 & 0.08 & 1.51 & 0.21 & 0.32 & 0.23 & 0.51 & : \\
\hline $\begin{array}{l}\text { Enfermedad } \\
\text { inflamatoria de los } \\
\text { órganos pélvicos } \\
\text { femeninos }\end{array}$ & 0.00 & 0.01 & 0.20 & 0.41 & 0.09 & 0.01 & 0.01 & 0.73 & 0.00 & 0.01 & 0.22 & 0.47 & 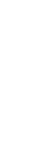 \\
\hline $\begin{array}{l}\text { Enfermedades } \\
\text { relacionadas con la } \\
\text { atención prenatal y el } \\
\text { parto }\end{array}$ & 0.01 & 0.02 & 1.00 & 0.51 & 0.00 & 0.00 & 0.00 & 1.55 & 0.03 & 0.05 & 1.82 & 0.90 & . \\
\hline Úlcera gástrica & 0.02 & 0.02 & 0.07 & 0.41 & 0.71 & 0.30 & 0.36 & 1.89 & 0.03 & 0.04 & 0.07 & 0.45 & $\frac{\cup}{\sigma}$ \\
\hline $\begin{array}{l}\text { Total de } \\
\text { hospitalizaciones } \\
\text { evitables }\end{array}$ & 6.69 & 2.78 & 3.11 & 5.87 & 8.85 & 4.33 & 4.63 & 36.27 & 6.90 & 5.44 & 4.36 & 7.13 & 这 \\
\hline
\end{tabular}


Quinquenio 2011-2015

Crecimiento promedio

Total

Grupos etarios

Total

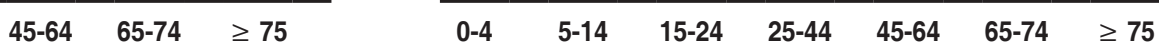

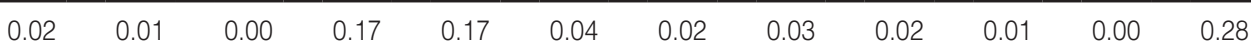

21.49

$\begin{array}{lll}0.18 & 0.06 \quad 0.05\end{array}$

$0.69 \quad 0.03$

$03 \quad 0.04$

$0.04 \quad 0.09$

$0.22 \quad 0.17$

0.05

0.04

0.63

$-4.89$

$0.51 \quad 0.24$

$0.33 \quad 6.41$

$1.96 \quad 1.38$

0.36

0.59

$\begin{array}{llll}0.44 & 0.20 & 0.28 & 5.21\end{array}$

$-3.13$

$\begin{array}{llll}0.03 & 0.01 & 0.02 & 0.12\end{array}$

$0.02 \quad 0.02$

$\begin{array}{llll}0.08 & 0.02 & 0.02 \quad 1.49\end{array}$

$49 \quad 0.59$

$\begin{array}{llll}0.12 & 0.08 & 0.14 & 1.00\end{array}$

$\begin{array}{lllll}0.20 & 0.06 & 0.04 & 2.01 & 0.91\end{array}$

$\begin{array}{lll}0.45 & 0.54 & 0.96\end{array}$

$96 \quad 4.69$

691.94

0.73

$\begin{array}{lllll}0.20 & 0.11 & 0.09 & 0.46 & 0.00\end{array}$

$\begin{array}{lllll}0.43 & 0.35 & 0.48 & 1.46 & 0.02\end{array}$

$\begin{array}{lllll}0.66 & 0.55 & 0.89 & 2.35 & 0.01\end{array}$

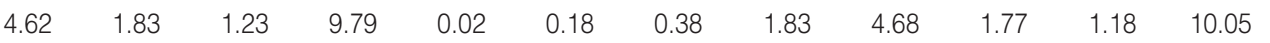

14.06

0.91

$\begin{array}{llll}0.12 & 0.03 & 0.03 & 1.20\end{array}$

$\begin{array}{llll}0.42 & 0.17 & 0.20 & 2\end{array}$

$\begin{array}{llll}0.49 & 0.14 & 0.12 \quad 2.02\end{array}$

$\begin{array}{llll}0.11 & 0.01 & 0.01 & 0.82\end{array}$

$0.82 \quad 0.00$

$0.37 \quad 0$.

$42 \quad 0.24$

$24 \quad 0.32$

32

0.14

$\begin{array}{lll}0.03 & 0.03 \quad 1.55\end{array}$

21.31

21.71

17.46

0.00 $\begin{array}{llll}0.00 & 0.00 \quad 2.80 \quad 0.02\end{array}$

$0.02 \quad 0.08$

2.55

1.18

$\begin{array}{llll}0.00 & 0.00 \quad 0.00 \quad 3.84\end{array}$

35.22

0.78

$0.36 \quad 0.47$

2.2

0.04

0.05

0.08

0.49

0.84

0.37

0.50

2.37

7.90

$10.17 \quad 5.01 \quad 5.60$

$44.60 \quad 7.29$

\begin{abstract}
5.76
\end{abstract}
5.35

\begin{abstract}
7.92
\end{abstract}
10.44

4.87

$5.61 \quad 47.24$

9.21 
Tabla 3. Listado de claves municipales según el Instituto Nacional de Estadística y Geografía y nivel de prioridad en uso hospitalario

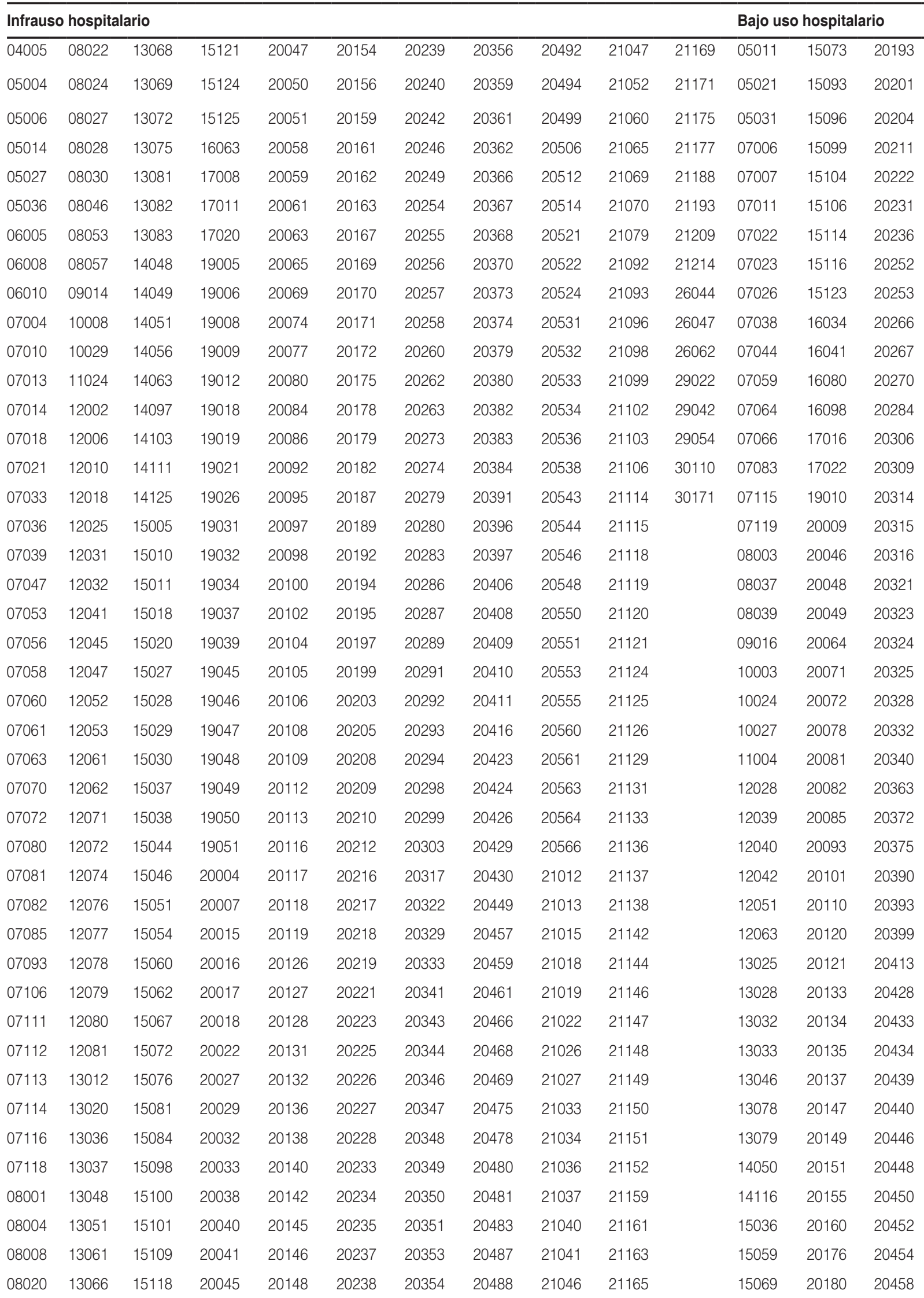


Alto uso hospitalario

\begin{tabular}{|c|c|c|c|c|c|c|c|c|c|c|c|c|c|c|}
\hline 20463 & 01002 & 01002 & 12070 & 19042 & 25017 & 32052 & 01003 & 07043 & 10004 & 14018 & 19015 & 20570 & 26067 & 29033 \\
\hline 20491 & 01010 & 01010 & 13001 & 20003 & 25018 & 32056 & 01004 & 07045 & 10007 & 14019 & 19016 & 21003 & 26070 & 29034 \\
\hline 20495 & 03002 & 03002 & 13003 & 20037 & 26015 & & 01006 & 07048 & 10012 & 14025 & 19020 & 21011 & 26071 & 29037 \\
\hline 20503 & 03003 & 03003 & 13006 & 20060 & 26050 & & 01007 & 07050 & 10014 & 14029 & 19023 & 21056 & 27001 & 29044 \\
\hline 20509 & 04002 & 04002 & 13015 & 20076 & 26057 & & 01008 & 07052 & 10018 & 14040 & 19027 & 21073 & 27002 & 29047 \\
\hline 20511 & 05001 & 05001 & 13019 & 20158 & 26066 & & 01009 & 07065 & 10020 & 14055 & 19035 & 21113 & 27003 & 30019 \\
\hline 20518 & 05028 & 05028 & 13030 & 20173 & 28001 & & 03001 & 07068 & 10028 & 14060 & 19036 & 21155 & 27004 & 30173 \\
\hline 20519 & 07003 & 07003 & 13043 & 20181 & 28010 & & 03009 & 07071 & 10032 & 14075 & 19040 & 21160 & 27005 & 30184 \\
\hline 20520 & 07034 & 07034 & 13047 & 20196 & 28011 & & 04003 & 07074 & 10036 & 14082 & 19044 & 21191 & 27006 & 30195 \\
\hline 20523 & 07035 & 07035 & 13054 & 20213 & 28021 & & 04004 & 07084 & 11001 & 14085 & 20001 & 23008 & 27007 & 31019 \\
\hline 20529 & 07042 & 07042 & 13055 & 20230 & 28032 & & 04006 & 07088 & 11002 & 14089 & 20006 & 23009 & 27008 & 31096 \\
\hline 20530 & 07054 & 07054 & 13057 & 20269 & 28033 & & 04007 & 07089 & 11003 & 14118 & 20019 & 23010 & 27010 & 32037 \\
\hline 20537 & 07055 & 07055 & 13073 & 20276 & 28035 & & 04009 & 07091 & 11014 & 15012 & 20020 & 25002 & 27011 & \\
\hline 20539 & 07099 & 07099 & 13084 & 20277 & 28037 & & 04010 & 07097 & 11015 & 15017 & 20026 & 25003 & 27012 & \\
\hline 20540 & 07104 & 07104 & 14024 & 20281 & 29001 & & 04011 & 07098 & 11016 & 15021 & 20031 & 25004 & 27013 & \\
\hline 20542 & 08007 & 08007 & 14037 & 20296 & 29005 & & 05002 & 07103 & 11026 & 15040 & 20035 & 25005 & 27014 & \\
\hline 20552 & 08018 & 08018 & 14038 & 20300 & 29008 & & 05003 & 07107 & 11028 & 15074 & 20042 & 25008 & 27017 & \\
\hline 20556 & 08059 & 08059 & 14042 & 20318 & 29012 & & 05007 & 07108 & 11029 & 15080 & 20073 & 25009 & 28003 & \\
\hline 20568 & 08064 & 08064 & 14062 & 20320 & 29014 & & 05009 & 07109 & 11030 & 15082 & 20123 & 25010 & 28006 & \\
\hline 21007 & 10001 & 10001 & 14064 & 20326 & 29021 & & 05012 & 07110 & 11031 & 15110 & 20177 & 25012 & 28007 & \\
\hline 21020 & 10006 & 10006 & 14077 & 20345 & 29030 & & 05015 & 08006 & 11034 & 15117 & 20190 & 25013 & 28008 & \\
\hline 21031 & 10011 & 10011 & 14081 & 20371 & 29038 & & 05019 & 08011 & 11037 & 17003 & 20191 & 25014 & 28012 & \\
\hline 21032 & 10017 & 10017 & 14092 & 20394 & 29046 & & 05020 & 08012 & 11041 & 17005 & 20250 & 25015 & 28014 & \\
\hline 21081 & 10021 & 10021 & 14094 & 20400 & 29055 & & 05024 & 08013 & 12008 & 17012 & 20295 & 26001 & 28015 & \\
\hline 21097 & 10025 & 10025 & 15009 & 20415 & 30074 & & 05025 & 08016 & 12012 & 17014 & 20311 & 26008 & 28017 & \\
\hline 21104 & 10031 & 10031 & 15105 & 20432 & 30138 & & 05032 & 08017 & 12034 & 17015 & 20313 & 26010 & 28019 & \\
\hline 21117 & 10035 & 10035 & 15107 & 20443 & 30185 & & 05033 & 08021 & 12035 & 17019 & 20337 & 26013 & 28022 & \\
\hline 21166 & 11018 & 11018 & 16011 & 20444 & 31008 & & 05037 & 08023 & 12055 & 17021 & 20338 & 26014 & 28024 & \\
\hline 21203 & 11025 & 11025 & 16014 & 20447 & 31021 & & 05038 & 08025 & 12066 & 18001 & 20364 & 26023 & 28025 & \\
\hline 21205 & 11035 & 11035 & 16024 & 20484 & 31057 & & 06002 & 08029 & 13029 & 18005 & 20369 & 26024 & 28026 & \\
\hline 21206 & 11038 & 11038 & 16056 & 20496 & 31061 & & 06006 & 08032 & 13031 & 18008 & 20378 & 26026 & 28031 & \\
\hline 26017 & 11039 & 11039 & 16071 & 20497 & 31065 & & 06007 & 08033 & 13040 & 18009 & 20387 & 26028 & 28040 & \\
\hline 29041 & 11042 & 11042 & 16076 & 20500 & 31085 & & 06009 & 08034 & 13053 & 18010 & 20419 & 26031 & 28043 & \\
\hline 29050 & 11044 & 11044 & 16081 & 21006 & 31102 & & 07002 & 08035 & 13058 & 18011 & 20431 & 26032 & 29003 & \\
\hline 29051 & 11046 & 11046 & 16099 & 21009 & 32014 & & 07008 & 08036 & 13059 & 18012 & 20435 & 26033 & 29006 & \\
\hline 29053 & 12005 & 12005 & 16103 & 21141 & 32020 & & 07009 & 08038 & 13060 & 18015 & 20436 & 26034 & 29007 & \\
\hline 29059 & 12013 & 12013 & 17017 & 21196 & 32022 & & 07016 & 08040 & 13077 & 18016 & 20442 & 26038 & 29009 & \\
\hline \multirow[t]{6}{*}{30201} & 12019 & 12019 & 17024 & 23005 & 32029 & & 07017 & 08043 & 14004 & 18018 & 20476 & 26040 & 29011 & \\
\hline & 12022 & 12022 & 17025 & 23007 & 32032 & & 07019 & 08048 & 14005 & 19002 & 20498 & 26041 & 29013 & \\
\hline & 12046 & 12046 & 17031 & 24007 & 32035 & & 07025 & 08050 & 14006 & 19003 & 20501 & 26042 & 29016 & \\
\hline & 12049 & 12049 & 18006 & 24044 & 32036 & & 07028 & 08052 & 14013 & 19007 & 20517 & 26048 & 29018 & \\
\hline & 12059 & 12059 & 19024 & 25006 & 32049 & & 07037 & 08058 & 14014 & 19011 & 20545 & 26053 & 29020 & \\
\hline & 12060 & 12060 & 19038 & 25011 & 32050 & & 07040 & 08067 & 14015 & 19014 & 20558 & 26063 & 29025 & \\
\hline
\end{tabular}

Sobreuso hospitalario 
a) Periodo 2001-2005

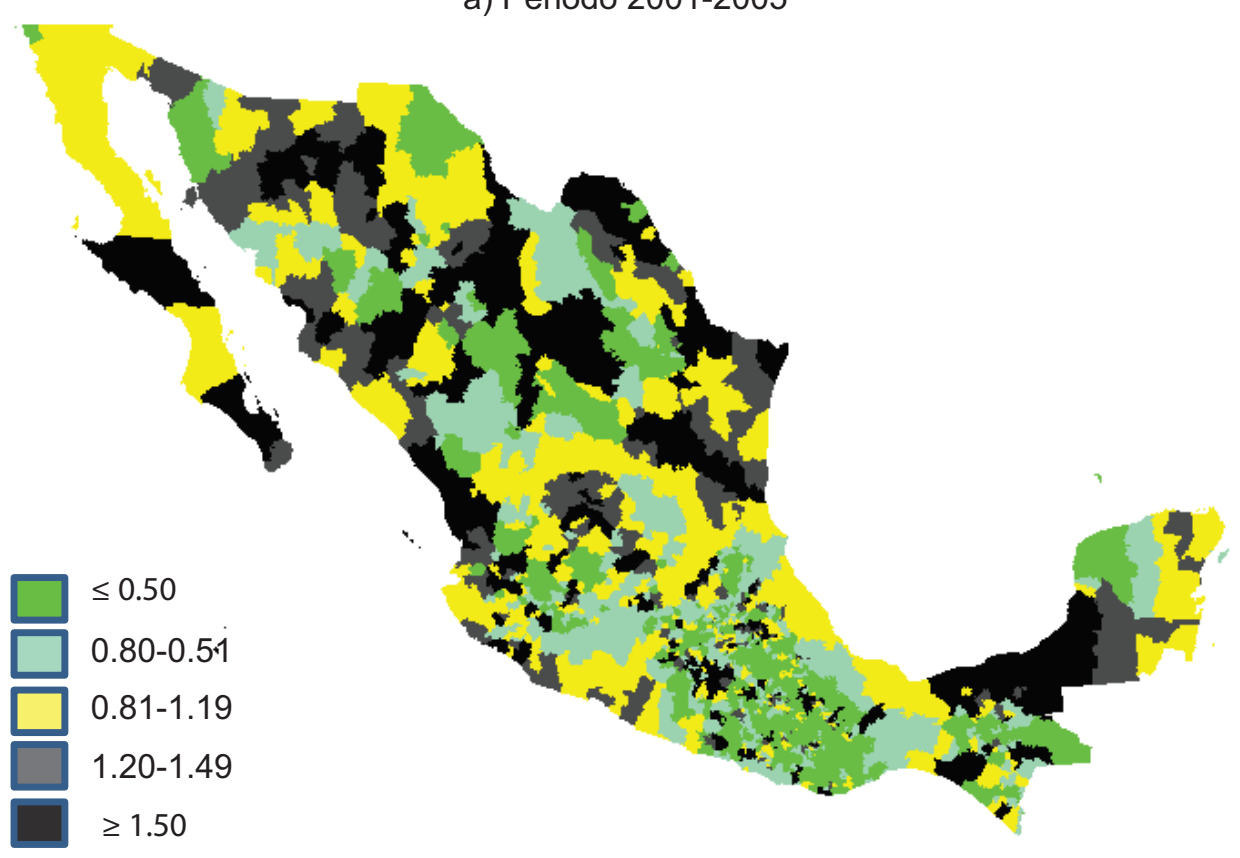

Figura 2. Prioridad de los municipios en función de los estratos de pertenencia según razones de hospitalizaciones evitables por quinquenios. México, 2001-2015.

Por causas de enfermedad según grupo etario, en los tres periodos se observó que en los grupos 0-4 y 5-14 la frecuencia se concentró en las enfermedades prevenibles por vacunación, gastroenteritis, deficiencias nutricionales, infecciones en oídos, nariz y garganta; neumonía bacteriana, asma y otras enfermedades de las vías respiratorias inferiores. En los grupos etarios entre 15 y 64 años ocurrieron con mayor frecuencia HE por condiciones evitables, anemia hipocrómica, hipertensión arterial, angina de pecho, diabetes mellitus, infección en el tracto urinario y del riñón; infección de la piel y del tejido subcutáneo, enfermedad inflamatoria de los órganos pélvicos femeninos, enfermedades relacionadas con la atención prenatal y el parto y úlcera gástrica. En los grupos de mayor edad, $\geq 65$ años, la insuficiencia cardiaca y las enfermedades cerebrovasculares fueron las causas más frecuentes de HE.

En la Tabla 2 se dispusieron las tasas estandarizadas por edad y sexo de HE según quinquenio, causa y grupo etario. Siguiendo el mismo orden de análisis de la Tabla 1, se observó incremento de $9.21 \%$ en promedio en la tasa nacional según el quinquenio: $36.27,44.60$ y 47.24 por 10000 habitantes.

La diabetes mellitus, la gastroenteritis y otras enfermedades de las vías respiratorias inferiores fueron las enfermedades con mayores tasas de HE en cada quinquenio. Las enfermedades cerebrovasculares ocuparon el cuarto lugar de mayor tasa en el primer quinquenio y fueron sustituidas por las enfermedades relacionadas con la atención prenatal y el parto en el segundo y último periodo.

Por causas de enfermedad se apreció incremento de las tasas a lo largo del periodo estudiado, excepto en las condiciones evitables, gastroenteritis y neumonía bacteriana, que tuvieron un leve descenso. Fueron ocho las causas que tuvieron un incremento superior a $10 \%$ : enfermedades relacionadas con la atención prenatal y el parto; infección en el tracto urinario y del riñón, enfermedades prevenibles por vacunación, epilepsia, infección de la piel y del tejido subcutáneo, anemia hipocrómica, otras enfermedades de las vías respiratorias inferiores e hipertensión arterial.

Por grupos de edad resaltaron en el total de egresos por HE los grupos 45-64, 25-44, 0-4 y $\geq 75$ años en los dos primeros quinquenios; en el tercer quinquenio, el grupo de 5-14 años sustituyó al $\geq 75$ años en la posición cuatro de los grupos con mayores tasas, aunque con poca diferencia.

Por causa y grupos de edad, considerando los cuatro valores más altos, el comportamiento de las tasas fue similar en cada quinquenio, con escasas variaciones. Se aprecia con claridad que en hipertensión arterial, angina de pecho, insuficiencia cardiaca, enfermedades cerebrovasculares, diabetes mellitus y 
úlcera gástrica, los grupos a partir de los 25 años tuvieron las tasas más altas; en las enfermedades prevenibles por vacunación, infecciones en oídos, nariz y garganta; epilepsia y enfermedades relacionadas con la atención prenatal y el parto las tasas más altas se ubicaron en los grupos etarios $\leq 25$ años. En el resto de las enfermedades se identificó mayor variabilidad en los grupos etarios con tasas altas de HE.

Los patrones geográficos de las razones de HE a nivel municipal observados en los mapas de los tres quinquenios tienen similitudes (Figura 1), con mayores semejanzas entre el segundo y tercer quinquenio. Resalta que existieron diversos puntos de concentración de alta o muy alta razón de HE a lo largo y ancho del país, cuyo centro se mantuvo fijo durante los tres quinquenios, a partir del cual se redujo o amplió la dimensión del conglomerado en función de los municipios que quedaron incluidos en los estratos superiores, por ejemplo, la parte sur de la costa del Golfo de México, que abarcó municipios costeros de Veracruz, Tabasco y Campeche, o municipios vecinos de los estados de Sinaloa y Nayarit, por mencionar algunas de las agrupaciones visibles en los estratos altos. Se observó lo mismo al atender el comportamiento espacial de los estratos bajos en los tres quinquenios, lo cual fue notorio en los municipios pertenecientes a Guerrero y Oaxaca.

En cuanto a la concentración de las HE en números absolutos, en el primer quinquenio los municipios que se ubicaron entre 20 y $49 \%$ por arriba de la tasa media de HE (187) $0 \geq 50 \%$ (294) concentraron $42.72 \%$ del total de HE (15.17 y $27.55 \%$, respectivamente); en el segundo quinquenio, en los municipios que se ubicaron en esas mismas estratificaciones por arriba de la tasa media de HE, 235 y 335 respectivamente, se concentró $43.04 \%$ de las HE (14.44 y $28.60 \%) ; y$ en el tercer quinquenio, en 301 y 452 municipios de acuerdo con la estratificación mencionada, se concentró $45.67 \%$ de las HE (11.74 y $33.93 \%$, respectivamente). Como puede observarse, en el estrato más alto de cada quinquenio se concentró el mayor número de $\mathrm{HE}$.

En la tabla de la Figura 1 pueden apreciarse los estadísticos de variabilidad geográfica de las razones de HE por municipio. En los tres quinquenios se observó alta variabilidad, más pronunciada en el primero, seguido por el segundo; el tercero fue el que tuvo mayor estabilidad. Las mayores variabilidades se identificaron en las razones de los percentiles (RVP) extremos de $1 / 99$ y $5 / 95$, que se redujeron importantemente en las RVP 10/90 y se aproximaron en los tres quinquenios en las RVP 20/80 y $25 / 75$, lo cual valida los cortes para la presentación de los datos en los mapas a partir de 20/80. El CVS indica alta variabilidad, ya que los valores cercanos a 1 fueron los esperados, lo que traduce que la incertidumbre se encuentra presente en el comportamiento espacial de las razones de $\mathrm{HE}$.

El mapa de municipios prioritarios por sobre o infrauso de hospitalizaciones también muestra similitud con los patrones geográficos descritos para cada quinquenio (Figura 2). El ejercicio dio como resultado 487 municipios prioritarios, 174 con alto uso del recurso hospitalario y 313 con sobreuso; entre ambos concentraron $35.83 \%$ de HE ocurridas en los tres quinquenios (8.58 5 y $27.25 \%$, respectivamente). En sentido inverso se identificaron 577 municipios prioritarios, 166 con bajo uso y 411 con infrauso, que apenas concentraron $6.91 \%$ de las HE en los tres periodos (2.81 y $4.10 \%$, respectivamente). En la parte media se concentraron 1353 municipios (en 41 municipios no se reportaron hospitalizaciones evitables durante el periodo), con $57.26 \%$ de las hospitalizaciones evitables.

Por último, en la Tabla 3 se enlistan las claves municipales de los municipios prioritarios, con sobre, alto, bajo e infrauso hospitalario. Las claves municipales pueden localizarse en el sitio web del INEGI.

\section{Discusión}

De acuerdo con nuestros resultados, las $\mathrm{HE}$ en la Secretaría de Salud oscilaron entre 9.26 y $10.39 \%$, similar a $10 \%$ reportado por Rodríguez Abrego et al. respecto a los servicios médicos del Instituto Mexicano del Seguro Social. En ambas instituciones, las causas más frecuentes de HE son diabetes mellitus, gastroenteritis, enfermedades respiratorias, enfermedades de la atención prenatal y parto y las infecciones de vías urinarias.

En la Secretaría de Salud, la tasa nacional de HE se ha incrementado en el periodo 2001-2015 de 36.27 a 47.24 por 10000 habitantes. Rodríguez Abrego et al. ${ }^{9}$ encontraron que la tasa nacional de HE había descendido de 87.5 por cada 10000 personas en 2001 a 72.6 en 2009. La discrepancia entre ambos estudios en cuanto a las tasas y su comportamiento radica principalmente en los periodos de estudio (2001-2015 versus 2001-2009), la fuente de egresos hospitalarios (Secretaría versus Instituto Mexicano del Seguro Social), las poblaciones diferentes para el cálculo de las tasas (total de población versus 
derechohabientes del Instituto Mexicano del Seguro Social) y el posible impacto de medidas que aplicó esa institución para reducir las HE.

En un estudio de 2011 sobre el desempeño hospitalario de la Secretaría de Salud ${ }^{8}$ se encontró que la tasa de HE fue de 29.6 por 10000 habitantes, incluyendo solo cinco enfermedades como referencia; es probable que si se hubieran considerado las 20 incluidas en el presente estudio, las cifras hubieran sido equiparables a las que se reportan.

Rodríguez Salgado et al..$^{10}$ encontraron que la tasa nacional de hospitalización por diabetes en 2014 fue de 14 hospitalizaciones por cada 10000 habitantes con edad $\geq 20$ años. Al considerar la condición de derechohabiencia, la tasa promedio de hospitalización para la población sin seguridad social fue de 15.3 y 12.0 por 10000 habitantes para la población con seguridad social. Nosotros encontramos una tasa de HE por diabetes de 7.85 en 2001-2005, 9.79 en 20062010 y 10.05 por 10000 habitantes entre 2011-2015. En la investigación de Rodríguez se calculó la tasa en un solo año en población con edad $\geq 20$ años y no fue estandarizada por edad ni sexo.

El estudio de las HE a través de la variabilidad geográfica de la práctica médica ofrece la oportunidad de identificar áreas de prioridad; en nuestra investigación se reconocieron municipios con infrauso o sobreuso del recurso hospitalario, tomando como referencia la media nacional. Esta división determinó que la población residente de esos municipios, según el caso, está recibiendo menos o más atención de la que necesita. Incluso, cabría la duda si la población con domicilio habitual en los municipios ubicados en la media o en los límites cercanos a ella considerados como aceptables, están recibiendo atención innecesaria, sobre todo si se consideran las características de las enfermedades que ocasionan HE: pueden ser prevenidas, diagnosticadas y tratadas en unidades médicas del primer nivel de atención primaria a la salud.

Los estudios ecológicos como el realizado tienen la limitación de no determinar las causas de la variabilidad de HE, por lo que en futuras investigaciones será necesario evaluarlas e integrar propuestas de solución que podrían ser disímiles y acordes con la multiplicidad de los factores involucrados en las diferentes regiones del país.

Una posible explicación podría ser que la incidencia o prevalencia de las enfermedades entre los municipios son distintas, sin embargo, la estandarización por edad y sexo indicó que esta posible razón tiene escaso poder aclarativo de la variabilidad encontrada entre municipios vecinos con poblaciones epidemiológicamente equivalentes.

Por lo anterior, tendría que recurrirse a la hipótesis de la incertidumbre, que establece como principales variables explicativas los problemas de accesibilidad, la oferta de servicios, la falta de guías clínicas o el no apego a los criterios consensuados para el manejo ambulatorio de las enfermedades potencialmente prevenibles.

Los problemas de accesibilidad pudieran explicar la concentración de municipios con bajo e infrauso en Guerrero, Oaxaca y Chiapas, donde se encuentran un número importante de hablantes de lenguas indígenas y el territorio es accidentado. ${ }^{18}$ En relación con la oferta de servicios, en Tabasco se encuentran municipios con sobreuso, al igual que en la parte sur de Veracruz o norte de Campeche; según datos de la Secretaría de Salud, entre 2001 y 2015, Tabasco fue el segundo estado con mayor número de consultorios, camas y médicos por cada 10000 habitantes, en primer lugar se encontró la Ciudad de México. ${ }^{19}$

En cuanto al no apego o desconocimientos de los criterios para el manejo de las patologías incluidas en el concepto de HE, un estudio efectuado en México señala que apenas $45 \%$ de los médicos aplicaba los criterios de las guías clínicas sectoriales para el manejo de las enfermedades crónico-degenerativas. ${ }^{20}$ Este último dato sugiere que la variabilidad nacional de HE obtenida en un contexto de bajo apego a la normatividad pudiera cambiar, como ha sucedidos en otros países ${ }^{21,22}$ o en el Instituto Mexicano del Seguro Social, ${ }^{9}$ donde constantemente se insiste en que la atención médica en el primer nivel se apegue a los criterios establecidos y que los pacientes reciban la atención hospitalaria realmente necesaria en relación con la evolución de la enfermedad y su control.,23,24

\section{Bibliografía}

1. Macinko J, Dourado I, Guanais FC. Enfermedades crónicas, atención primaria y desempeño de los sistemas de salud: diagnóstico, herramientas e intervenciones. EEUU: Banco Interamericano de Desarrollo; 2011.

2. Rivero-Serrano O. Factores que han modificado la práctica médica. Gac Med Mex. 2002;138:331-346.

3. Muenchberger $\mathrm{H}$, Kendall E. Predictors of preventable hospitalization in chronic disease: Priorities for change. J Public Health Policy. 2010; 31:150-163.

4. Caminal-Homar J, Morales-Espinoza M, Sánchez Ruiz E, Cubells-Larrosac MJ, Bustins-Poblet M.Hospitalizaciones prevenibles mediante una atención primaria oportuna y efectiva. Aten Primaria 2003;31:6-17.

5. Rosano A, Abo-Loha Ch, Falvo R, van der Zee J, Ricciardi W, Guasticchi $\mathrm{G}$, et al. The relationship between avoidable hospitalization and accessibility to primary care: a systematic review. Eur J Public Health. 2012;23:356-360.

6. Ridao-López M, García-Armesto S, Peiró S, Bernal-Delgado E. Evaluación de la variabilidad geográfica de la práctica médica como herramienta de evaluación de políticas sanitarias. Presupuesto y Gasto Público. 2012;68:193-210. 
7. Desempeño hospitalario. México: Secretaría de Salud; 2003.

8. Observatorio del desempeño hospitalario 2011. México: Secretaría de Salud; 2011.

9. Rodríguez-Abrego G, Zurita-Garza B, Ramírez-Sánchez T, Herrera-Rojas JJ. Serie sobre hospitalizaciones evitables y fortalecimiento de la atención primaria en salud. El caso de México. México: Banco Interamericano de Desarrollo; 2012.

10. Rodríguez-Salgado M, Fernández-Cantón SB, Rizo-Amézquita JN, Jiménez-Silva AA. Hospitalizaciones evitables por diabetes como condición sensible a la atención ambulatoria en las principales instituciones públicas de salud de México. Boletín Conamed-OPS. 2017;2(10). Disponible en: http://www.dgdi-conamed.salud.gob.mx/ojs-conamed/index.php/ BCCCSP/article/view/572/850

11. Secretaría de Salud. [Sitio web]. Acciones y programas. Disponible en https://www.gob.mx/salud/archivo/acciones_y_programas

12. Secretaría de Salud. [Sitio web]. Egresos hospitalarios. [Consultado 2017 Jul 29]. Disponible en: http://www.dgis.salud.gob.mx/contenidos/ basesdedatos/std_egresoshospitalarios_gobmx.html

13. Alfradique ME, Bonolo PF, Dourado I, Lima-Costa MF, Macinko J, Mendonça CS, et al. Internações por condições sensíveis à Atenção Primária: a construção da lista brasileira como ferramenta para medir o desempenho do sistema de saúde (Projeto ICSAP - Brasil). Cad Saúde Publica. 2009;25:1338-1339.

14. Centro Nacional de Excelencia Tecnológica en Salud. [Sitio web]. Guías de práctica clínica. [Consultado 2017 Jul 29]. Disponible en: http://cenetec-difusion.com/gpc-sns/?cat $=52$

15. Consejo Nacional de Población. Proyecciones de población por municipio. [Consultado 2017 Jul 29]. Disponible en: http://www.conapo.gob.mx/ es/CONAPO/Proyecciones
16. Propín-Frejomil E. Teorías y métodos en geografía económica. México: Universidad Nacional Autónoma de México/Instituto de Geografía; 2003.

17. Instituto Nacional de Estadística y Geografía. Mapa digital de México. [Consultado 2017 Jul 29]. Disponible en: http://www.inegi.org.mx/geo/ contenidos/mapadigital

18. Censo de población y vivienda 2010. México: Instituto Nacional de Estadística y Geografía; 2013.

19. Secretaría de Salud. Recursos en salud. [Consultado 2017 Jul 29]. Disponible en: http://www.dgis.salud.gob.mx/contenidos/basesdedatos/estandar_gobmx.html

20. Poblano-Verástegui O, Vieyra-Romero WI, Galván-García AF, Fernández-Elorriaga M, Rodríguez-Martínez Al, Saturno-Hernández PJ. Calidad y cumplimiento de guías de práctica clínica de enfermedades crónicas no transmisibles en el primer nivel. Salud Publica Mex. 2017;59:165-175.

21. Librero J, Ibañez-Beroiz B, Peiró S, Ridao-López M, Rodríguez-Bernal CL, Gómez-Romero FJ, et al. Trends and area variations in Potentially Preventable Admissions for COPD in Spain (2002-2013): a significant decline and convergence between areas. BMC Health Serv Res. 2016; 16:367-376

22. Angulo-Pueyo E, Natalia Martínez-Lizaga N, Ridao-López M, García-Armesto S, Bernal-Delgado E. Evolución de las hospitalizaciones potencialmente evitables por condiciones crónicas en España. Gac Sanit. 2016;30:52-54.

23. Bernal-Delgado E, García-Armesto S, Campillo-Artero C. ¿Sirven los estudios de variabilidad geográfica de la práctica para informar la desinversión? Varias cautelas y algunas reflexiones. Gac Sanit. 2013;27:7-11.

24. Gutiérrez-Alba G, González-Block MA, Reyes-Morales H. Desafíos en la implantación de guías de práctica clínica en instituciones públicas de México: estudio de casos múltiple. Salud Publica Mex. 2015;57:547-554. 